\title{
Indirect effects of COVID-19 in referring women to gynecologic oncology, perinatology and gynecology clinics in Iran
}

\author{
Zahra Khazaeipour $^{1}$ (D) $\cdot$ Erfan Razavi $^{1} \cdot$ Mohammad-Taha Pahlevan-Fallahy $^{1}$
}

Received: 5 January 2021 / Accepted: 11 May 2021 / Published online: 31 May 2021

(c) The Author(s), under exclusive licence to Springer-Verlag GmbH Germany, part of Springer Nature 2021

\begin{abstract}
Purpose COVID-19 has captured the world. We hypothesized that this pandemic reduced referral of other non-COVID-19 patients to the hospitals or clinics, including gynecological and perinatological referrals. Women can be at risk in limited use of health services.

Methods In this retrospective study, referrals from gynecologic oncology, perinatology, and gynecology clinics in a large teaching hospital of Tehran University of Medical Sciences (TUMS) were compared from February 20 to May 20, 2020, with the same period in 2019. Finally, referral trends in 2020 were compared with the COVID-19 admission pattern.

Results Total admissions to all three clinics declined 63\% in 2020 compared to 2019. There was a significant relationship between the number of visits to three clinics during these 2 years $(p<0.001)$. The reduction in referrals to the gynecology clinic was more than gynecologic oncology and perinatology. The COVID-19 referral pattern was conversely linked to gynecology-related admissions.

Conclusion As the pandemic situation makes patients hesitant to go to the hospitals or not, health policymakers should consider other non-COVID issues, including maternal and fetal concerns. Providing safe places for other patients to visit is a goal that can be achieved through developing guidelines for nosocomial hygiene and training informed healthcare staff. Moreover, non-urgent visits should be avoided or postponed. This issue calls for new strategies, including telemedicine in situations similar to the current pandemic to both identify and manage such conditions.
\end{abstract}

Keywords Women's health $\cdot$ Health policy $\cdot$ COVID-19 pandemic $\cdot$ Perinatology $\cdot$ Gynecology

\section{Introduction}

Since December 2019, millions of people have been infected with the new type of coronavirus known as severe acute respiratory syndrome coronavirus 2 (SARS-CoV-2) [1]. The potential for person-to-person transmission of SARS-CoV-2 has led to an exponential rise in the number of coronavirus cases in 2019 (COVID-19) worldwide [2]. On March 11, 2020, the World Health Organization (WHO) officially declared the public health emergency as a pandemic [3].

The COVID-19 pandemic has indirectly affected other disorders, including obstetrics and gynecology-related conditions. Apart from mortality, COVID-19 has affected

Zahra Khazaeipour

khazaeipour@tums.ac.ir

Brain and Spinal Cord Injury Research Center, Neuroscience Institute, Tehran University of Medical Sciences, Tehran, Iran women's health in several ways. For instance, the prevention, diagnosis, and treatment of malignancies, including gynecologic oncology, have been changed dramatically during the pandemic [4-6]. Besides, although childbearing women have not suffered higher mortality rates from COVID-19, inadequate access to healthcare service and unsupervised childbirth would indirectly lead to more complications and deaths $[7,8]$. This issue is similar to the experience of the Ebola epidemic in Sierra Leone [9]. Moreover, gynecology admissions for a routine check-up might have decreased due to social distancing and lockdown measures implemented by governments or fear of contact with COVID-19-infected patients or healthcare staffs.

Several studies have been conducted to address nonCOVID disease referrals during the COVID-19 era. However, few studies have examined only gynecology admissions [10-14]. The number of admissions in three Italian hospitals for gynecological emergencies has decreased significantly due to the COVID-19 lockdown 
[10]. Furthermore, Frey et al. reported that about $38 \%$ of women experienced cancellation, postponing, or modification in their scheduled gynecologic oncology care due to COVID-19 [11]. In a single study in Israel, childbearing women postponed their referral to the delivery room and emergency departments during the COVID-19 pandemic compared to the previous year [13].

There is an urgent need to discuss gynecologic oncology, perinatology, and gynecology referral trends in Iran. First, most of the available evidence is only related to the number of gynecological admissions during the pandemic, while the overall pattern of admissions has not got enough attention. Second, few studies have been specifically dedicated to determining the effect of the pandemic on different gynecology-related clinic admissions. Third, no study has addressed gynecological admissions in Iran.

This study seeks the notion of describing the admission trends of gynecologic oncology, perinatology, and gynecology clinics in a large teaching hospital of Tehran University of Medical Sciences (TUMS) in Tehran from February 20 to May 20, 2020 compared with the corresponding 3 months in 2019.

\section{Materials and methods}

This was a retrospective study conducted at the large teaching hospital of TUMS. From the information technology (IT) department of this hospital, we retrieved the records of women referring to three clinics (Gynecologic Oncology, Perinatology, and Gynecology clinics) as well as COVID-19 visits to the mentioned hospital. The collected data on patient referrals from February 20 to May 20 in 2020 were compared with the corresponding period in 2019. The starting day of study was established as the first day of the month based on the Iranian date. No inclusion and exclusion criteria were defined. Data were recorded from an electronic database and analyzed using SPSS software version 22 (Statistical Package of Social Science, Chicago, IL, USA). Numbers and frequencies were reported for overall referrals to the aforementioned clinics in 2020 and 2019. Weekly descriptive statistics were reported as the number of gynecology-related or COVID-19 referrals. Moreover, the weekly trends of admissions were illustrated in line charts drawn by Microsoft Excel 2016 software. Chi-square analysis was used to examine the differences between the two qualitative variables (referrals to clinics in 2 years). $P$ value $<0.05$ was considered statistically significant.

This study was ethically approved by the Research Ethics Committee of TUMS (IR.TUMS.VCR.REC.1399.075) on March 30, 2020.

\section{Results}

In this study, the numbers of gynecologic oncology, perinatology, and gynecology visits have been evaluated throughout the first 3 months of the pandemic (from February 20 to May 20, 2020). In 2020, overall admission to all three clinics was $63 \%$ lower than in 2019 during the study period (2092 versus 5627). The difference between the number of visits to the three clinics during the 2 years was significant $(p<0.001)$, the highest decline was in the gynecology clinic (Table 1; Fig. 1).

\section{Trends of weekly admissions to gynecology-related clinics in 2020 and 2019}

The referral trend indicates that since February 20, the number of referrals in 2020 has decreased compared to 2019. The decline in referrals to the clinics of gynecologic oncology and perinatology continued until March 19 (after which the clinics were closed because of the Iranian New Year holidays), but it was until March 5 in the gynecology clinic, because there were no referrals after March 5 (Fig. 2).

From March 20 to 26, the clinics were closed due to the Iranian New Year holidays. The clinics reopened on March 26 , and there was an immediate return to gynecologic oncology and perinatology. However, referrals were lower in 2020 than in 2019, and admissions increased steadily in 2020 (Fig. 2b, c). In the case of the gynecological clinic, the number of referrals increased from April 16 and with a gentle slope compared to the previous year (Fig. 2c).

\section{The overall admission pattern in 2020 compared to 2019 and COVID-19 referral}

Figure 3 indicates the total number of referrals in all three clinics over 2 years. The increase in admissions was moderate after March 26, 2020 but increased sharply the previous year.

Table 1 Admissions to gynecology-related clinics

\begin{tabular}{llll}
\hline Clinic & \multicolumn{2}{l}{ Admission year } & Decrease rate \\
\cline { 2 - 3 } & 2019 & 2020 & \\
\hline Gynecologic oncology & $1803(69.4 \%)$ & $796(30.6 \%)$ & $-55.9 \%$ \\
Perinatology & $2947(71.8 \%)$ & $1159(28.2 \%)$ & $-60.7 \%$ \\
Gynecology & $877(86.5 \%)$ & $137(13.5 \%)$ & $-84.4 \%$ \\
Total & $5627(72.9 \%)$ & $2092(27.1 \%)$ & $-62.8 \%$ \\
\hline
\end{tabular}

$P<0.001$

The frequency of admissions to gynecologic oncology, perinatology, and gynecology clinics in the year 2020 compared with 2019 
Fig. 1 Admissions to gynecology-related clinics. The number of admissions to gynecologic oncology, perinatology, and gynecology clinics in the year 2020 compared with 2019

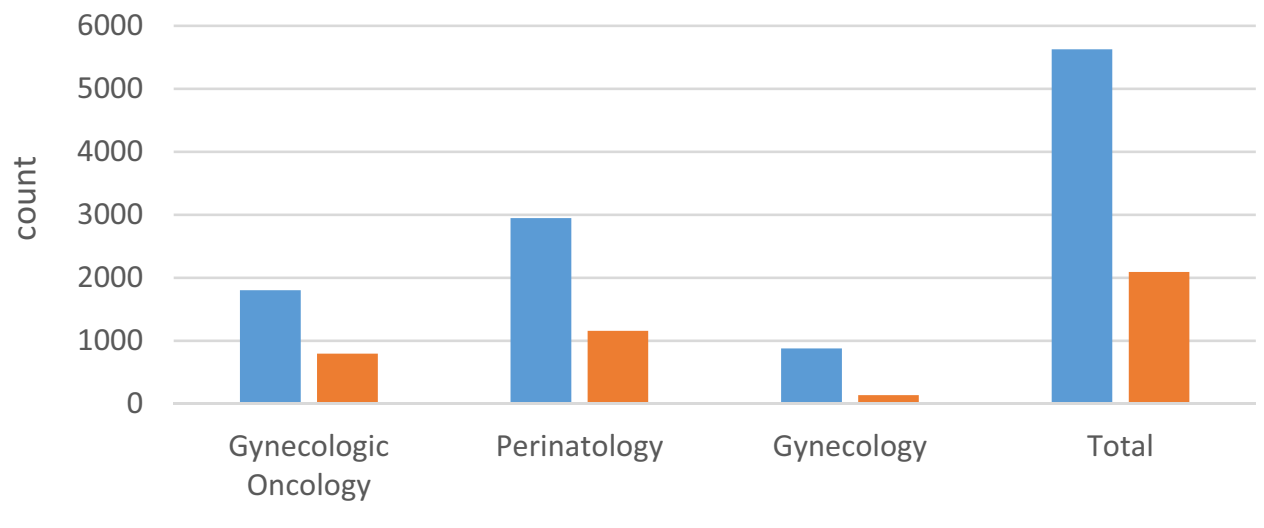

clinic
The trend of referrals to clinics was inversely related to the trend of COVID-19 patients admitted to the hospital in 2020; with the decrease of COVID-19 patients, the trend of referrals increased (Fig. 4).

\section{Discussion}

In recent years, numerous crises and epidemics have challenged health systems, including the emergence of infectious diseases such as Ebola, severe acute respiratory syndrome (SARS), and the flu, along with other natural disasters. In the winter of 2019, the SARS-COV-2 outbreak and the rapid expansion of COVID-19 patients around the world posed a serious challenge in terms of financial and human resources to health systems that were not prepared to face a pandemic, for example, a surge in the number of patients will require more hospital beds and ICU care beyond the capacity of the health care system. To slow down the further spread of the virus, people were strongly advised to stay home and keep their social distance and, in some countries, strict quarantine laws have been placed to prevent people from becoming more infected. This situation raised a question for many patients, whether they should visit the hospitals or not. As a result, various reasons such as fear of infection, commuting restrictions due to strict quarantine policies, limitations in resource allocation in hospitals and job burnouts made healthcare seekers and providers encounter problems and patients started either going to the hospital later, when the severity of the disease has increased or not referring at all, which can make their condition worse or reduce the effectiveness of their treatment [15-20].

In the field of gynecologic oncology, perinatology and gynecology, women also reduced their hospital visits; they either delayed hospitalization, or visited the hospital due to emergencies such as childbirth. On one hand, due to the higher risk of COVID-19 in these people, fewer visits help prevent further spread of the viral disease, and on the other hand, it might lead to delays in the necessary treatment and care, which can be dangerous to their health. For example, women should receive prenatal care and cancer screening and, if necessary, they should be treated at the best time; otherwise, it can have serious consequences for the fetus and mother [21, 22]. A study demonstrated that women are more likely to choose home births in India, because they are afraid of becoming infected if they refer to hospitals and strict lockdown laws and fear of infection forced them not to visit hospitals [7]. Another study revealed that Italian women asked their general practitioner or private gynecologist for home visits to have a safer environment and to avoid going to the hospital [23]. The COVID-19 pandemic has been shown to increase stress and anxiety in childbearing women, which may lead to increased preterm labor and preeclampsia [24]. It has also been observed that excessive use of detergents and sanitizers may cause skin irritations and make people feel unsafe about going to the hospital [24].

This study showed a sharp decrease in the number of women referring to the obstetrics and gynecology department in 2020 compared to the corresponding period in 2019. The number of women receiving care in the gynecologic oncology, perinatology, and gynecology clinics had a significant decrease, even more than $50 \%$ in some time periods. Studies have shown that the rate of ectopic pregnancy in the normal population is nearly $2 \%$ and the incidence of genetic disorders and congenital malformations is nearly $3-5 \%$ which necessitates the use of proper maternal care, which has proven to be significantly useful in reducing pregnancy complications [25-27]. Consequently, delaying or skipping pregnancy tests and checkups might reduce the risk of contamination, but the consequences can be severe [19, 20].

Different solutions such as increasing the capacity of intensive care unit (ICU) have been implemented to solve 
Fig. 2 Referrals to gynecologyrelated clinics. The trends of referrals to the gynecologic oncology (A), perinatology (B), and gynecology $(\mathbf{C})$ clinics in the year 2019 and 2020
A

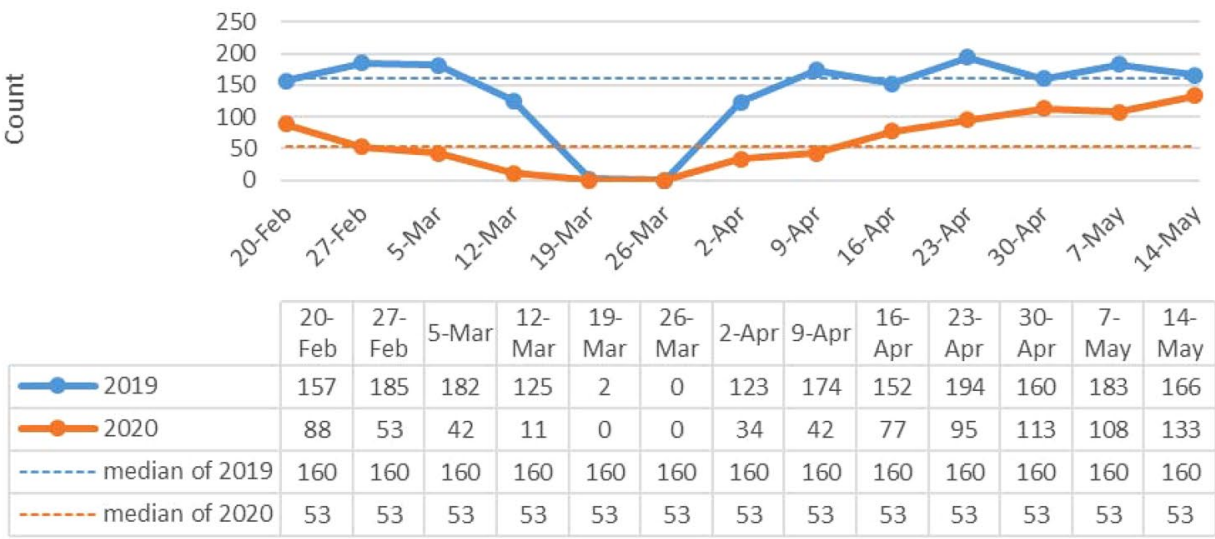

$\longrightarrow 2019 \longrightarrow 2020 \quad------$ median of $2019 \quad------$ median of 2020

B

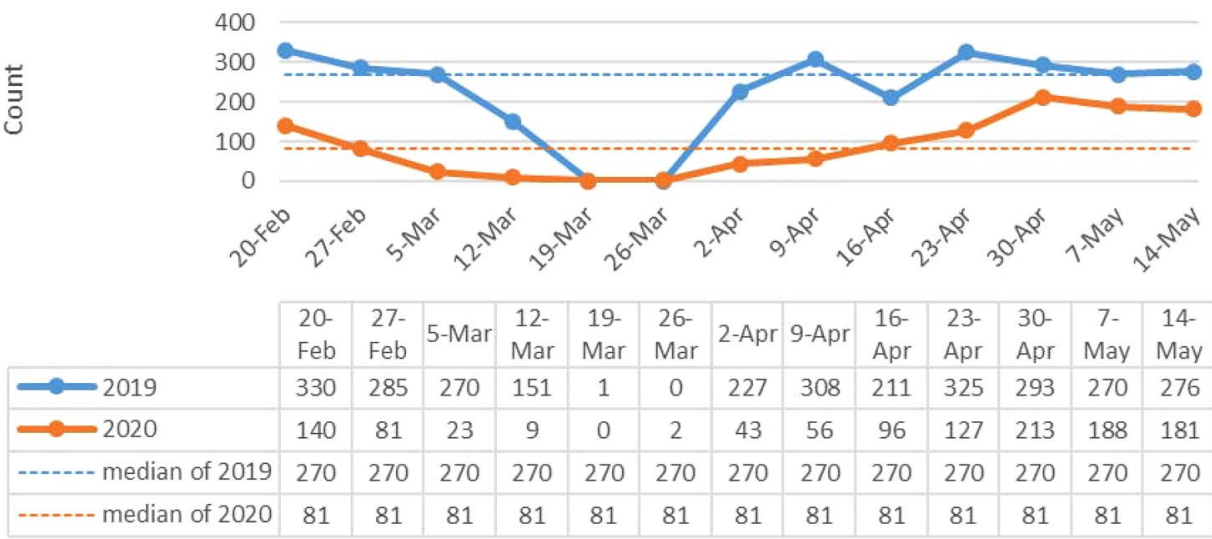

$\longrightarrow 2019 \longrightarrow 2020 \quad------$ median of $2019 \quad$------ median of 2020

C

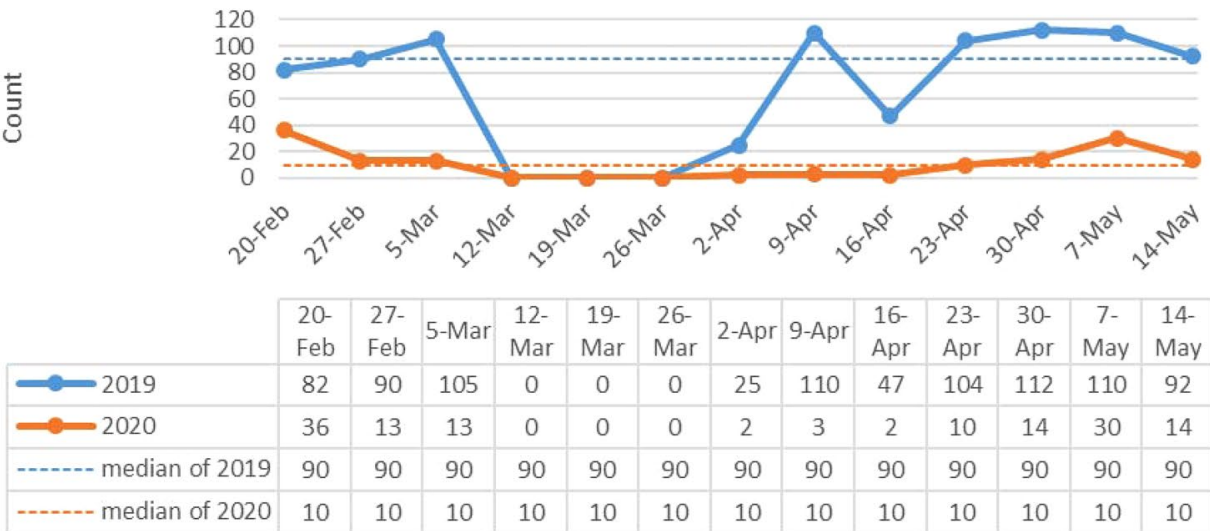




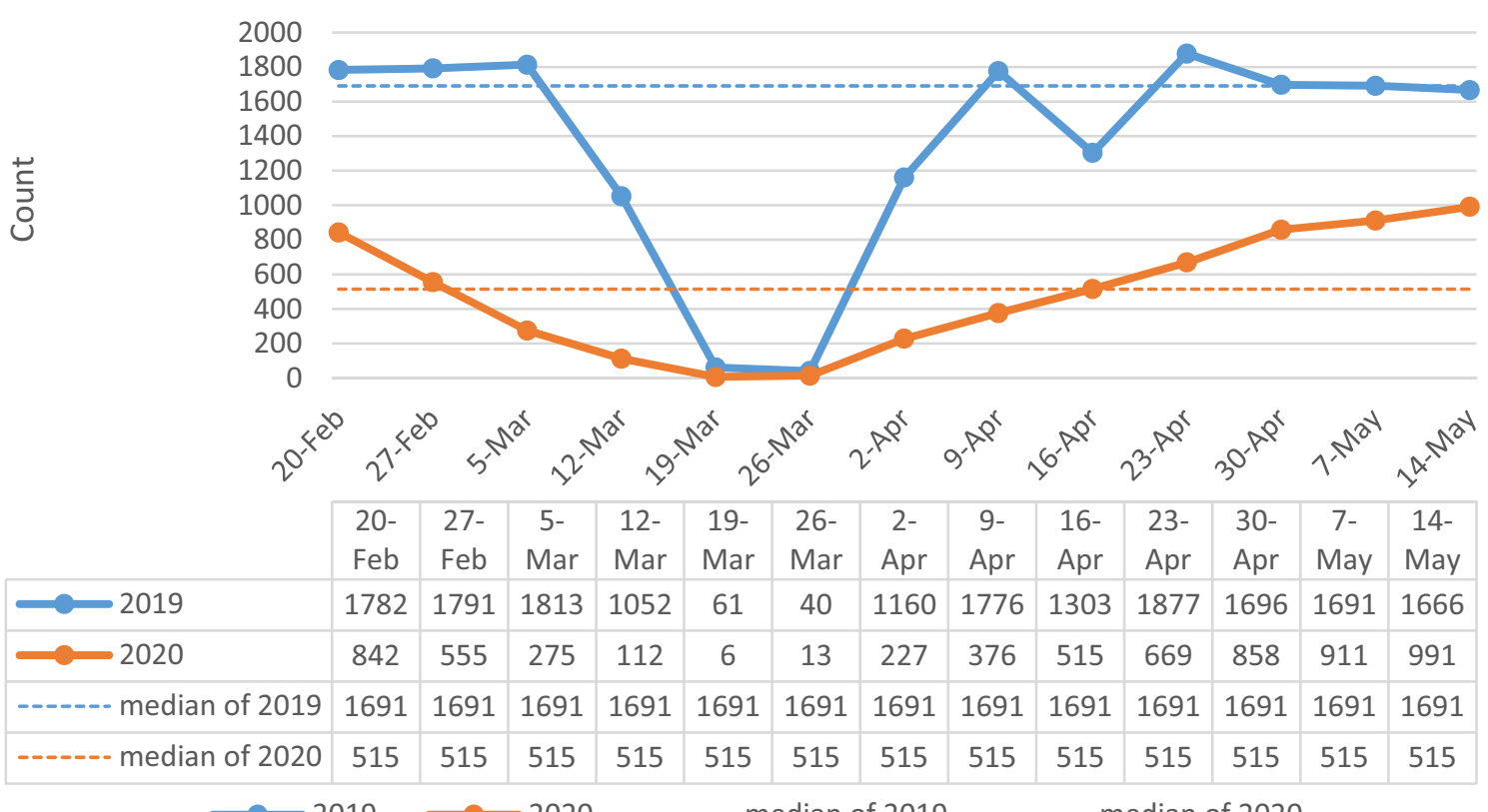

$-2019$

Fig. 3 Total referrals. Trends of overall referrals to the gynecologic oncology, perinatology, and gynecology clinics in the year 2019 and 2020

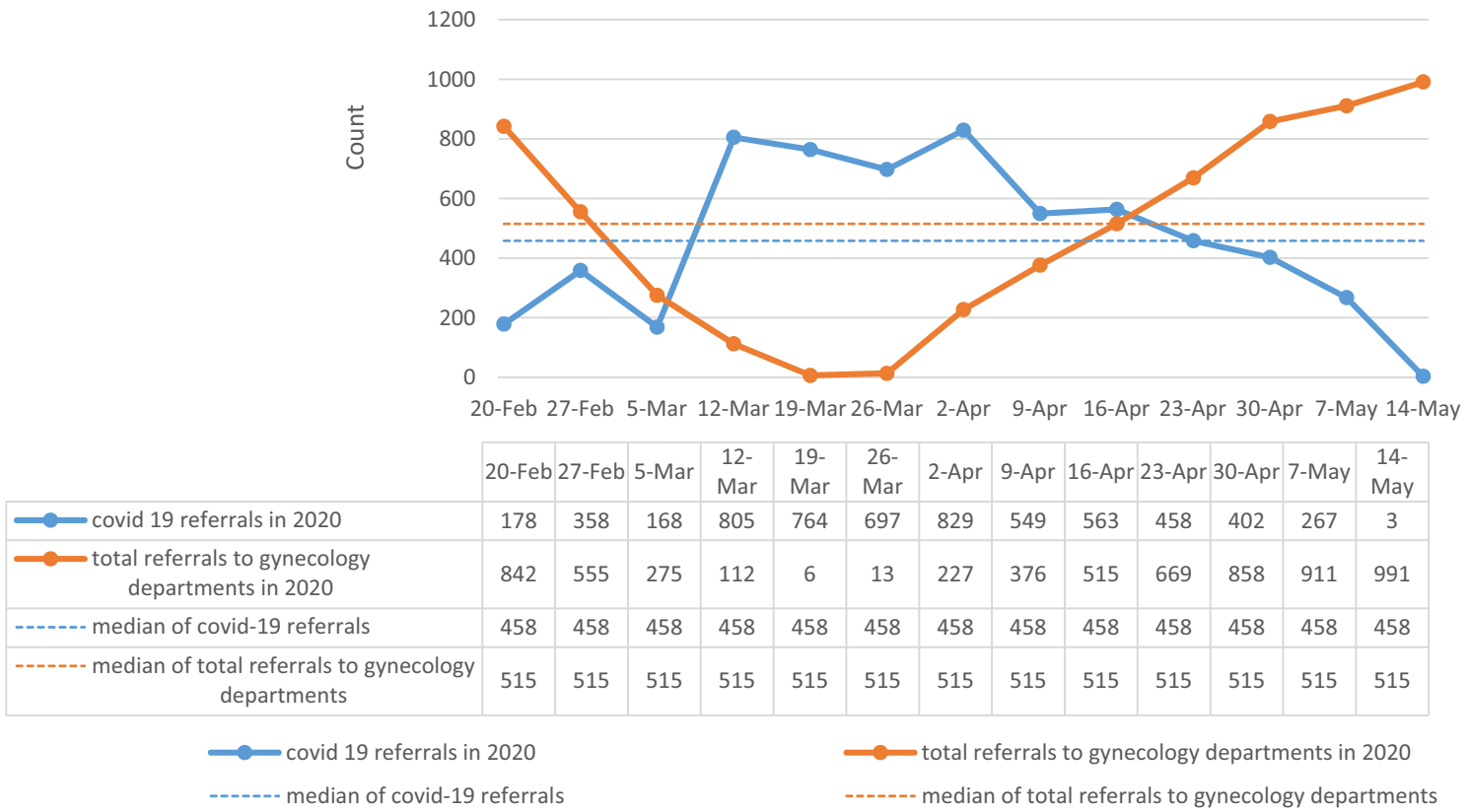

Fig. 4 COVID-19 and overall Gynecology referrals in 2020: The comparison between trends in COVID-19 admissions and overall referrals related to gynecology

this issue. Some hospitals around the world have begun to adapt to this critical situation using telemedicine and online counseling to decrease the risk of contamination [28-37]. Telemedicine allows healthcare providers to consult patients, relieve their stress and clear any misunderstanding that might have confused their minds and help them to communicate effectively. Some studies suggested that using education, training and publishing protocols can improve hospital care while preparing the healthcare workers for critical situations and reducing fear among people [37-39]. In a study, a protocol consisting of risk level-based patient triage, use of improved infection control protocols, care planning, and 
admission control was used [37]. Reducing unnecessarily planned elective operations proved to be a useful solution in the short term $[37,39]$. Because of the epidemic, governments should encourage people not to delay hospital visits for emergencies as they plan to reduce unnecessary gatherings at the same time.

Our study showed that patients in gynecologic oncology and perinatology clinics, who need continuous and timely care, continued to visit in urgent conditions, and as the number of COVID-19 patients decreased, their number of visits increased.

However, the number of women referred to the gynecology clinic has decreased significantly and did not increase so much even with the decline in the number of COVID-19 patients. It seems that women who referred to this clinic had fewer emergency conditions or could solve their problems with remote medical advice. Perhaps in these circumstances, some additional and unnecessary referrals of people may have also decreased.

For some diseases, it is possible for the physicians to examine the patients remotely and give them the necessary advice and only in urgent cases, to ask them to go to the clinic or hospital. In this way, unnecessary referrals can be prevented. The state of the COVID-19 pandemic caused changes in the healthcare system that may continue even after the pandemic is over.

Implications for practice and policy: In the present study, we aim to get health planners and policymakers to consider improving health systems in epidemics and pandemics. Other cases and diseases that require constant, complete, and timely treatment, such as pregnant women and gynecological diseases, should not be ignored in pandemics.

The hygiene principles and infection control protocols should be ensured in medical centers and hospitals. Healthcare workers and patients should be trained in infection prevention and personal hygiene. The transmission rate of infectious diseases is sometimes very high and its complications have to be taken seriously. In addition, it is recommended to find solutions to avoid unnecessary visits to hospitals and medical centers.

\section{Limitations}

Our study is a single-centered retrospective study with a number of limitations. Though unlikely, there is the possibility of the decreased number of women in our center being compensated by other hospitals or clinics. We also do not have information regarding the number of home births and other outpatient birth clinics which could help to clarify this issue. Despite being a single-centered study, our data which were obtained from a great teaching hospital of TUMS information technology center might reflect the overall situation, but large-scale national studies are required to make sure of the generalizability of the results of this study. Further follow-up in the period after the COVID-19 pandemic is recommended to make sure that the numbers of women return to the previously known numbers before the COVID-19 outbreak. The clinical consequences of the decrease in women's visit rates are unknown and more studies are needed before further conclusions can be drawn.

Further data analysis, such as assessing mortality rates of gynecology-related conditions and referral statistics to other hospitals, especially those not involved in the admission and treatment of COVID-19 patients, may provide better information on the consequences caused by the pandemic.

This was a descriptive study, and the ultimate application goal of our program is to reduce the complications of indirect effects of COVID-19 pandemic or any other pandemic that people may suffer in the future. Of course, this is not achieved by the current descriptive study alone. The present study mainly seeks the notion of raising concerns about women's health during the pandemic as well as to provide a basis for future research.

\section{Conclusion}

The pandemic situation has caused more stress and anxiety in women, making it difficult for them to go to the hospital due to health concerns or not. Eventually, fear of contamination led to a decrease in the number of women visiting hospitals, which could have severe consequences. Necessary steps should be taken in hospitals to reduce the risk of transmission of infection to women during outbreaks. The staff should be adequately trained in the conditions that should be considered when going to a hospital.

People should also be educated on how and when to visit hospitals to prevent their conditions from getting worse. Also, hospitals should improve their infection control protocols and provide the necessary equipment to reduce the risk of infection for both women who receive healthcare services and healthcare workers.

Acknowledgements We thank vice president of research of Imam Khomeini Hospital Complex and information technology department of given hospital for their assistance in data collection.

Author contributions Zahra Khazaeipour: conceptualization, methodology, validation, formal analysis, writing - original draft, writing-review and editing supervision, visualization, supervision, project administration. Erfan Razavi; formal analysis, writing-original draft, writing - review and editing, visualization. Mohammad-Taha Pahlevan-Fallahy: formal analysis, writing — original draft, writingreview and editing, visualization.

Funding This research did not receive any specific grant from funding agencies in the public, commercial, or not-for-profit sectors. 
Availability of data and materials Corresponding author had full access to all the data in the study and takes responsibility for the integrity of the data and the accuracy of the data analysis.

\section{Declarations}

Conflict of interest The authors declare having no conflict of interest related to this work.

Ethical approval This study was ethically approved by the Research Ethics Committee of Tehran University of Medical Sciences (IR. TUMS.VCR.REC.1399.075) on 30 March 2020.

Informed consent We could not get informed consent, because the study was retrospective and we had electronic patient records. However, patient's information was confidential and all ethical standards were followed.

\section{References}

1. Lai CC, Shih TP, Ko WC, Tang HJ, Hsueh PR (2020) Severe acute respiratory syndrome coronavirus 2 (SARS-CoV-2) and coronavirus disease-2019 (COVID-19): The epidemic and the challenges. Int J Antimicrob Agents 55(3):105924

2. Mohapatra RK, Pintilie L (2020) The recent challenges of highly contagious COVID-19, causing respiratory infections: Symptoms, diagnosis, transmission, possible vaccines, animal models, and immunotherapy. Chem Biol Drug Des 96(5):1187-1208

3. Cucinotta D, Vanelli M (2020) WHO declares COVID-19 a pandemic. Acta Biomed 91(1):157-160

4. Martinelli F, Garbi A (2020) Change in practice in gynecologic oncology during the COVID-19 pandemic: a social media survey. Int J Gynecolog Cancer 30(8):1101-1107

5. Ramirez PT, Chiva L (2020) COVID-19 global pandemic: options for management of gynecologic cancers. Int J Gynecol Cancer 30(5):561-563

6. Bogani G, Brusadelli C (2020) Gynecologic oncology at the time of COVID-19 outbreak. Gynecol Oncol 31(4):e72

7. Goyal M, Singh P, Singh K, Shekhar S, Agrawal N, Misra S (2020) The effect of the COVID-19 pandemic on maternal health due to delay in seeking health care: Experience from a tertiary center. Int J Gynaecol Obstet. https://doi.org/10.1002/ijgo.13457

8. Sahin D, Tanacan A, Erol SA, Anuk AT, Eyi EGY, Ozgu-Erdinc AS, Yucel A, Keskin HL, Tayman C, Unlu S et al (2020) A pandemic center's experience of managing pregnant women with COVID-19 infection in Turkey: A prospective cohort study. Int J Gynaecol Obstet 151(1):74-82

9. Sochas L, Channon AA, Nam S (2017) Counting indirect crisisrelated deaths in the context of a low-resilience health system: the case of maternal and neonatal health during the Ebola epidemic in Sierra Leone. Health Policy Plann 32(3):iii32-iii39

10. Grandi G, Del Savio MC, Caroli M, Capobianco G, Dessole F, Tupponi G, Petrillo M, Succu C, Paoletti AM, Facchinetti F (2020) The impact of COVID-19 lockdown on admission to gynecological emergency departments: Results from a multicenter Italian study. Int J Gynaecol Obstet 151(1):39-42

11. Frey MK, Fowlkes RK, Badiner NM, Fishman D, Kanis M, Thomas C, Christos PJ, Martin P, Gamble C, Balogun OD et al (2020) Gynecologic oncology care during the COVID-19 pandemic at three affiliated New York City hospitals. Gynecol Oncol 159(2):470-475
12. Kasaven LS, Saso S, Barcroft J, Yazbek J, Joash K, Stalder C, Ben Nagi J, Smith JR, Lees C, Bourne T et al (2020) Implications for the future of obstetrics and gynaecology following the COVID-19 pandemic: a commentary. Int J Gynaecol Obstet 127(11):1318-1323

13. Kugelman N, Lavie O, Assaf W, Cohen N, Sagi-Dain L, Bardicef M, Kedar R, Damti A, Segev Y (2020) Changes in the obstetrical emergency department profile during the COVID-19 pandemic. J Maternal-fetal Neonatal Med 10(1080/14767058):1847072

14. Khalil A, von Dadelszen P, Draycott T, Ugwumadu A, O'Brien $\mathrm{P}$, Magee L (2020) Change in the incidence of stillbirth and preterm delivery during the COVID-19 pandemic. JAMA 324(7):705-706

15. De Filippo O, D'Ascenzo F, Angelini F, Bocchino PP, Conrotto F, Saglietto A, Secco GG, Campo G, Gallone G, Verardi R (2020) Reduced rate of hospital admissions for ACS during Covid-19 outbreak in Northern Italy. New Engl J Med 383:88-89

16. Kansagra AP, Goyal MS, Hamilton S, Albers GW (2020) Collateral effect of Covid-19 on stroke evaluation in the United States. New Engl J Med 383:400-401

17. Baum A, Schwartz MD (2020) Admissions to Veterans affairs hospitals for emergency conditions during the COVID-19 pandemic. JAMA 324:96-99

18. Meyer R, Levin G, Hendin N, Katorza E (2020) Impact of the COVID-19 outbreak on routine obstetrical management. Isr Med Assoc J 22(8):483-488

19. Griffin I, Benarba F, Peters C, Oyelese Y, Murphy T, Contreras D, Gagliardo C, Nwaobasi-Iwuh E, DiPentima MC, Schenkman A (2020) The impact of COVID-19 infection on labor and delivery, newborn nursery, and neonatal intensive care unit: prospective observational data from a single hospital system. Am J Perinatol 37(10):1022-1030

20. Grandi G, Del Savio MC, Caroli M, Capobianco G, Dessole F, Tupponi G, Petrillo M, Succu C, Paoletti AM, Facchinetti F (2020) The impact of COVID-19 lockdown on admission to gynecological emergency departments: Results from a multicenter Italian study. Int J Gynaecol Obstet 151:39-42

21. Schwartz DA, Graham AL (2020) Potential maternal and infant outcomes from (Wuhan) Coronavirus 2019-nCoV infecting pregnant women: lessons from SARS, MERS, and other human coronavirus infections. Viruses 12(2):194

22. Vanni G, Pellicciaro M, Materazzo M, Bruno V, Oldani C, Pistolese CA, Buonomo C, Caspi J, Gualtieri P, Chiaravalloti A et al (2020) Lockdown of breast cancer screening for COVID-19: possible scenario. Vivo 34(5):3047-3053

23. Dell'Utri C, Manzoni E, Cipriani S, Spizzico C, Dell'Acqua A, Barbara G, Parazzini F, Kustermann A (2020) Effects of SARS Cov-2 epidemic on the obstetrical and gynecological emergency service accesses. What happened and what shall we expect now? Eur J Obstet Gynecol Reprod Biol 254:64-68

24. Fakari FR, Simbar M (2020) Coronavirus pandemic and worries during pregnancy; a letter to editor. Arch Acad Emerg Med 8(1):e21-e 21

25. Obstetricians ACo, Gynecologists (2018) ACOG Practice Bulletin No 193: tubal ectopic pregnancy. Obstet Gynecol 131(3):91-103

26. Carroli G, Rooney C, Villar J (2001) How effective is antenatal care in preventing maternal mortality and serious morbidity? An overview of the evidence. Paediatr Perinat Epidemiol 15:1-42

27. CfD C (2008) Prevention: Update on overall prevalence of major birth defects-Atlanta, Georgia, 1978-2005. MMWR Morb Mortal Wkly Rep 57(1):1

28. Ohannessian R, Duong TA, Odone A (2020) Global telemedicine implementation and integration within health systems to fight the COVID-19 pandemic: a call to action. JMIR Public Health Surveill 6(2):18810 
29. Bokolo Anthony J (2020) Use of telemedicine and virtual care for remote treatment in response to COVID-19 pandemic. J Med Syst 44(7):132

30. Boehm K, Ziewers S, Brandt MP, Sparwasser P, Haack M, Willems F, Thomas A, Dotzauer R, Höfner T, Tsaur I et al (2020) Telemedicine online visits in urology during the COVID-19 pandemic-potential, risk factors, and patients' perspective. Eur Urol 78(1):16-20

31. Bedford J, Enria D, Giesecke J, Heymann DL, Ihekweazu C, Kobinger G, Lane HC, Memish Z, Oh MD, Sall AA et al (2020) COVID-19: towards controlling of a pandemic. Lancet (London, England) 395(10229):1015-1018

32. Fryer K, Delgado A, Foti T, Reid CN, Marshall J (2020) Implementation of obstetric telehealth during COVID-19 and beyond. Matern Child Health J 24(9):1104-1110

33. Grimes CL, Balk EM, Crisp CC, Antosh DD, Murphy M, Halder GE, Jeppson PC, Weber LeBrun EE, Raman S, Kim-Fine S et al (2020) A guide for urogynecologic patient care utilizing telemedicine during the COVID-19 pandemic: review of existing evidence. Int Urogynecol J 31(6):1063-1089

34. Schinköthe T, Gabri MR, Mitterer M, Gouveia P, Heinemann V, Harbeck N, Subklewe M (2020) A web- and app-based connected care solution for COVID-19 In- and outpatient care: qualitative study and application development. JMIR Public Health Surveill 6(2): 19033

35. Wu H, Sun W, Huang X, Yu S, Wang H, Bi X, Sheng J, Chen S, Akinwunmi B, Zhang CJP et al (2020) Online antenatal care during the COVID-19 pandemic: opportunities and challenges. J Med Internet Res 22(7):e19916

36. Aziz A, Zork N, Aubey JJ, Baptiste CD, D’Alton ME, Emeruwa UN, Fuchs KM, Goffman D, Gyamfi-Bannerman C, Haythe JH et al (2020) Telehealth for high-risk pregnancies in the setting of the COVID-19 pandemic. Am J Perinatol 37(8):800-808

37. Zhou F, Yu T, Du R, Fan G, Liu Y, Liu Z, Xiang J, Wang Y, Song B, Gu X et al (2020) Clinical course and risk factors for mortality of adult inpatients with COVID-19 in Wuhan, China: a retrospective cohort study. Lancet (London, England) 395(10229):1054-1062

38. Harvey S, Zalud I (2020) Obstetric hospital preparedness for a pandemic: an obstetric critical care perspective in response to COVID-19. J Perinat Med 48(9):874-882

39. Wurmb T, Scholtes K, Kolibay F, Schorscher N, Ertl G, Ernestus RI, Vogel U, Franke A, Kowalzik B (2020) Hospital preparedness for mass critical care during SARS-CoV-2 pandemic. Crit Care 24(1):386

Publisher's Note Springer Nature remains neutral with regard to jurisdictional claims in published maps and institutional affiliations. 